\title{
Die Haar- und Haargruppendichtigkeit bei den Mischlingsfeten.
}

\author{
Von \\ Toshiko Iidaka \\ Aus dem Anatomischen Institut der Keio Universität, Tokyo. \\ (Direktor: Prof. Dr. T. Taniguchi).
}

Mit 7 Texttabellen.

In unserem Institut sind ausgedehnte Untersuchungen über die Zahlenverhältnisse der Haare (Haarbälge) der verschiedenen Körperteile bei den verschiedenen Tierarten (Mogi, u. Yoshioka), Menschenrassen (Taniguchi u. Shibayama), Altersstufen nach der Geburt (Taniguchi und Shibayama), bei den japanischen Embryonen (Kato) schon unternommen worden. Neuerdings habe ich einige Mischlingsfeten zwischen Japanerinnen einerseits und Europäern und Neger anderseits bekommen. Es war mir deshalb eine sehr erwünschte Aufgabe, die Befunde bei den Mischlingsfeten mit den bei den japanischen Feten von Kato miteinander $\mathrm{zu}$ vergleichen.

Für meine Untersuchung habe ich die Hautstücke von 6 Mischlingsfeten, wie in der Tabelle 1 gezeigt, verwandt.

Tabelle 1. Material.

\begin{tabular}{|c|c|c|c|c|}
\hline Fall & Mischlingsfeten & & Geschlecht & Körpergröße \\
\hline 1 & Jap. $\times$ Europ. & 5 & 9 & $24.0 \mathrm{~cm}$ \\
\hline 2 & Jap. $\times$ Europ. & 6 & $\delta$ & 28.0 \\
\hline 3 & Jap. × Europ. & 7 & $\delta$ & 36.0 \\
\hline 4 & Jap. × Europ. & 8 & ᄋ & 42.0 \\
\hline 5 & Jap. $\times$ Neger & 8 & \& & 42.0 \\
\hline 6 & Jap. $\times$ Europ. & 10 & $\hat{\delta}$ & 54.0 \\
\hline
\end{tabular}

Jap. $=$ Japanerin, Europ. $=$ Europäer. 
Alle Leichen wurden mit Formol-Alkohollösung im ganzen Körper injiziert und dann in 10\% Formalinlösung konserviert. Die Hautstücke wurden sorgfältig herausgeschnitten, in Zelloidin eingebettet und in Flächenschnittserien von $40 \mu$ Dicke zerlegt. Die Färbung geschah mit Hämatoxylin (Hansen)-Eosin. Folgende 5 Körperteile wurden beobachtet. 1. Scheitel 2. Brust 3. Rücken 4. Oberarm (Beugeseite) 5. Unterschenkel (Streckseite).

Die Haarbälge in den oberflächlichen Schnitten wurden in 30 facher Vergrößerung auf ein Papierstück projektiert gezeichnet. Dann zählte ich die auf ein bestimmtes Quadrat $(100 \mathrm{qcm})$ dieses Papiers gezeichneten Haarbälge und Haarbalggruppen, wiederholte also diese Zählung in 10 verschiedenen Hautteilen der einzelnen Körperteile, addierte dann die gewonnene $Z$ ahl und rechnete den Mittelwert aus, den ich dann auf $1 \mathrm{qcm}$ Hautflächeninhalt umrechnete.

Im Folgenden werde ich meine Befunde tabellarisch angeben. (Tab. 2.-Tab. 7).

Die Haarzahl in $1 \mathrm{qcm}$ Hautfächeninhalt.

Die Haarzahl in $1 \mathrm{qcm}$ Hautflächeninhalt bei dem 5 monatigen Fetus ist, wie Tabelle 2 zeigt, nach den Körperteilen verschieden. Sie ist am Scheitel $(1927,8)$ am größten, dann folgen die Brust $(1249,3)$, der Beugeseite des Oberarms und am geringsten ist sie an Streckseite des Uniterschenkels.

Die Haarzahl bei dem 6 monatigen Fetus schwankt auch in den verschiedenen Körperteilen bedeutend. Sie ist am Scheitel (2275) am

Tabelle 2. 5 monatiger Mischlingsfetus (Japanerin $\times$ Europäer).

\begin{tabular}{|c|c|c|c|c|c|}
\hline \multirow{2}{*}{ Körperteile } & \multicolumn{4}{|c|}{ Haar- und Haargruppenzahl in 1 qcm und Prozentsatz } \\
\cline { 2 - 6 } & Haar & \multicolumn{4}{|c|}{ Haargruppe } \\
\cline { 2 - 6 } & 1927.8 & $\begin{array}{c}572.4 \\
(483 \%)\end{array}$ & $\begin{array}{c}486 \\
(40.9 \%)\end{array}$ & $\begin{array}{c}127.8 \\
(10.8 \%)\end{array}$ & 1186.2 \\
\hline Scheitel & 1249.3 & $\begin{array}{c}932.4 \\
(85.5 \%)\end{array}$ & $\begin{array}{c}153 \\
(14.2 \%)\end{array}$ & $\begin{array}{c}3.6 \\
(0.3 \%)\end{array}$ & 1089 \\
\hline Brust & 1006.6 & $\begin{array}{c}947 \\
(97.1 \%)\end{array}$ & $\begin{array}{c}27.1 \\
(2.7 \%)\end{array}$ & $\begin{array}{c}1.8 \\
(0.2 \%)\end{array}$ & 975.9 \\
\hline Rücken & 932.4 & $\begin{array}{c}865.8 \\
(96.6 \%)\end{array}$ & $\begin{array}{c}19.8 \\
(2.2 \%)\end{array}$ & $\begin{array}{c}9 \\
(1.2 \%)\end{array}$ & 894.6 \\
\hline Oberarm (Bs) & 742.5 & $\begin{array}{c}706.5 \\
(97.5 \%)\end{array}$ & $\begin{array}{c}18 \\
(2.5 \%)\end{array}$ & 724.5 \\
\hline Unterschenkel & (Ss) & & & \\
\hline
\end{tabular}

HG = Haargruppe, Bs= Beugeseite, $\mathrm{Ss}=$ Streckseite. 
Die Haar- und Haargruppendichtigkeit bei den Mischlingsfeten

Tabelle 3. 6-monatiger Mischlingsfetus (Japanerin $\times$ Europäer)

\begin{tabular}{|c|c|c|c|c|c|c|c|c|c|}
\hline \multirow{3}{*}{ Körperteile } & \multicolumn{9}{|c|}{ Haar- und Haargruppenzahl in $1 \mathrm{qcm}$ und Prozentsatz } \\
\hline & \multirow{2}{*}{ Haar } & \multicolumn{8}{|c|}{ Haargruppe } \\
\hline & & $1-\mathrm{HG}$ & $2-\mathrm{HG}$ & $3-\mathrm{H} / \mathrm{s}$ & 4-HG & $5-\mathrm{HG}$ & $6-\mathrm{HG}$ & 7-HG & Summe \\
\hline Scheitel & 2275 & $\left|\begin{array}{c}216 \\
(22.4 \%)\end{array}\right|$ & $\begin{array}{c}401.4 \\
(41.7 \%)\end{array}$ & $\begin{array}{c}201.6 \\
(20.9 \%)\end{array}$ & $\begin{array}{c}90 \\
(9.3 \%)\end{array}$ & $\left(\begin{array}{c}32.4 \\
3.4 \%\end{array}\right)$ & $\left(\begin{array}{c}19.8 \\
2.1 \%\end{array}\right.$ & $\left(\begin{array}{c}1.8 \\
0.2 \%\end{array}\right)$ & 963 \\
\hline Brust & 1004.4 & $\begin{array}{c}250.2 \\
(39.9 \%\end{array}$ & $\begin{array}{c}374.4 \\
(59.7 \%)\end{array}$ & $\left(\begin{array}{c}1.8 \\
0.4 \%\end{array}\right)$ & & & & & 626.4 \\
\hline Rücken & 1032.3 & $\begin{array}{c}260.1 \\
(40.3 \%)\end{array}$ & $\begin{array}{c}383.4 \\
(59.4 \%)\end{array}$ & $\left(\begin{array}{c}1.8 \\
(0.3 \%)\end{array}\right.$ & & & & & 645.3 \\
\hline $\begin{array}{l}\text { Oberarm } \\
\text { (Bs) }\end{array}$ & 714.6 & $\begin{array}{c}433.8 \\
(75.6 \%)\end{array}$ & $\begin{array}{c}140.4 \\
(24.4 \%)\end{array}$ & & & & & & 574.2 \\
\hline $\begin{array}{l}\text { Unter- } \\
\text { schenkel(Ss) }\end{array}$ & 869.4 & $\mid \begin{array}{c}448.2 \\
(70.1 \%)\end{array}$ & $\begin{array}{c}151.2 \\
(23.6 \%)\end{array}$ & $\left(\begin{array}{c}39.6 \\
(6.2 \%)\end{array}\right.$ & & & & & 639 \\
\hline
\end{tabular}

Tabelle 4. 7-monatiger Mischlingsfetus (Japanerin $\times$ Europäer).

\begin{tabular}{|c|c|c|c|c|c|c|c|c|c|}
\hline \multirow{3}{*}{ Körperteile } & \multicolumn{9}{|c|}{ Haar- und Haargruppenzahl in $1 \mathrm{qcm}$ und Prozentsatz } \\
\hline & \multirow{2}{*}{ Haar } & \multicolumn{8}{|c|}{ Haargruppe } \\
\hline & & & & & & & & & \\
\hline Scheitel & 1825.2 & $\begin{array}{l}36 \\
(6.1 \%)\end{array}$ & $\begin{array}{c}136.8 \\
(23.2 \%)\end{array}$ & $\begin{array}{c}244.8 \\
(41.4 \%)\end{array}$ & $\begin{array}{c}118.8 \\
(20.1 \%)\end{array}$ & $\left(\begin{array}{c}27 \\
4.6 \%\end{array}\right)$ & $\left(\begin{array}{c}18 \\
(3.1 \%)\end{array}\right.$ & $\left(\begin{array}{c}9 \\
1.5 \%\end{array}\right)$ & 590.4 \\
\hline Brust & 1035.8 & $\begin{array}{c}219.6 \\
36.0 \%)\end{array}$ & $\begin{array}{c}340.2 \\
(56.0 \%)\end{array}$ & $\left(\begin{array}{c}48.6 \\
(8.0 \%)\end{array}\right.$ & & & & & 608.4 \\
\hline Rücken & 803.3 & $\begin{array}{c}217.8 \\
(42.6 \%)\end{array}$ & $\begin{array}{c}289.8 \\
(56.7 \%)\end{array}$ & $\left(\begin{array}{c}3.6 \\
0.7 \%)\end{array}\right.$ & & & & & 511.2 \\
\hline $\begin{array}{l}\text { Oberarm } \\
\text { (Bs) }\end{array}$ & 10922 & $\begin{array}{c}194.4 \\
(33.4 \%)\end{array}$ & $\left|\begin{array}{c}318.6 \\
(54.6 \%)\end{array}\right|$ & $\begin{array}{c}70.2 \\
(12.0 \%)\end{array}$ & & & & & 583.2 \\
\hline $\begin{array}{l}\text { Unter- } \\
\text { schenkel(Ss) }\end{array}$ & 865.8 & $\begin{array}{c}315 \\
(58.5 \%)\end{array}$ & $\begin{array}{c}120.6 \\
(22.4 \%)\end{array}$ & $\mid \begin{array}{c}100.8 \\
(18.7 \%)\end{array}$ & $\left(\begin{array}{c}1.8 \\
0.4 \%)\end{array}\right.$ & & & & 538.2 \\
\hline
\end{tabular}

größten, dann kommen der Rücken $(1032,3)$, die Brust (1004,4), die Streckseite des Unterschenkels (869,4), und an der Streckseite des Oberarms $(714,6)$ ist sie am geringsten.

Am Scheitel und an der Streckseite des Unterschenkels ist die Haarzahl beim 6 monatigen Fetus größer als beim 5 monatigen.

Auch bei dem 7 monatigen Fetus weist der Scheitel $(1825,2)$ die höchste Haarzahl auf, dann kommen der Oberarm $(1092,2)$, die Brust $(1035,8)$, der Oberarm und sie ist am Unterschenkel $(865,8)$ am geringsten. 
T. Iidaka

Tabelle 5. 8-monatiger Mischlingsfetus (Japanerin $\times$ Europäer).

\begin{tabular}{|c|c|c|c|c|c|c|c|c|c|c|}
\hline \multirow{3}{*}{$\begin{array}{l}\text { Körper- } \\
\text { teile }\end{array}$} & \multicolumn{10}{|c|}{ Haar- und Haargruppenzahl in $1 \mathrm{qcm}$ und Prozentsatz } \\
\hline & \multirow{2}{*}{ Haar } & \multicolumn{9}{|c|}{ Haargruppe } \\
\hline & & & & & & & & & 1 & \\
\hline Scheitel & 1427.2 & $\begin{array}{c}77.2 \\
(15.9 \%)\end{array}$ & $\begin{array}{c}106.2 \\
(21.9 \%)\end{array}$ & $\begin{array}{c}176.4 \\
(36.4 \%)\end{array}$ & $\begin{array}{c}57.6 \\
(11.1 \%)\end{array}$ & $\begin{array}{c}34.2 \\
(7.1 \%)\end{array}$ & $\begin{array}{c}21.6 \\
(4.4 \%)\end{array}$ & $\begin{array}{c}9 \\
(1.8 \%)\end{array}$ & $\left(\begin{array}{c}1.8 \\
(0.4 \%)\end{array}\right.$ & 484 \\
\hline Brust & 703.8 & $\begin{array}{c}160.2 \\
(38.3 \%)\end{array}$ & $\begin{array}{c}228.6 \\
(54.8 \%)\end{array}$ & $\begin{array}{c}28.8 \\
(6.9 \%)\end{array}$ & & & & & & 417.6 \\
\hline Rücken & 410.4 & $\begin{array}{c}133.2 \\
(49.1 \%)\end{array}$ & $\begin{array}{c}138.6 \\
(50.9 \%)\end{array}$ & & & & & & & 271.8 \\
\hline $\begin{array}{l}\text { Oberarm } \\
\text { (Bs) }\end{array}$ & 511.2 & $\begin{array}{c}158.4 \\
(49.4 \%)\end{array}$ & $\begin{array}{c}136.8 \\
(42.7 \%)\end{array}$ & $\left(\begin{array}{c}21.6 \\
6.8 \%\end{array}\right)$ & $\begin{array}{l}3.6 \\
(1.1 \%)\end{array}$ & & & & & 320.4 \\
\hline $\begin{array}{l}\text { Unter- } \\
\text { schenkel } \\
\text { (Ss) }\end{array}$ & 684 & $\begin{array}{c}82.8 \\
(26.5 \%)\end{array}$ & $\begin{array}{c}84.6 \\
(27.2 \%)\end{array}$ & $\begin{array}{c}144 \\
(46.3 \%)\end{array}$ & & & & & & 311.4 \\
\hline
\end{tabular}

Tabelle 6. 8-monatiger Mischlingsfetus (Japanerin $\times$ Neger).

\begin{tabular}{|c|c|c|c|c|c|c|c|c|}
\hline \multirow{3}{*}{ Körperteile } & \multicolumn{8}{|c|}{ Haar- und Haargruppenzahl in $1 \mathrm{qcm}$ und Prozentsatz } \\
\hline & \multirow{2}{*}{ Haar } & \multicolumn{7}{|c|}{ Haagruppe } \\
\hline & & $1-\mathrm{HG}$ & $2-\mathrm{HG}$ & $3-\mathrm{HG}$ & $4-\mathrm{HG}$ & 5-HG & $6-\mathrm{HG}$ & \\
\hline Scheitel & 1238.2 & $\begin{array}{c}109.8 \\
(19.3 \%)\end{array}$ & $\begin{array}{l}284.4 \\
(50 \%)\end{array}$ & $\begin{array}{c}127.8 \\
(22.5 \%)\end{array}$ & $\left.\begin{array}{c}37.8 \\
6.6 \%\end{array}\right)$ & $\left(\begin{array}{c}9 \\
(1.6 \%\end{array}\right)$ & & 568.8 \\
\hline Brust & 670.6 & $\begin{array}{c}126 \\
(36.2 \%)\end{array}$ & $\begin{array}{c}219.6 \\
(63.3 \%)\end{array}$ & $\left(\begin{array}{c}1.8 \\
0.5 \%\end{array}\right)$ & & & & 347.4 \\
\hline Rücken & 705.6 & $\begin{array}{c}216 \\
(46.8 \%)\end{array}$ & $\begin{array}{c}244.8 \\
(53.2 \%)\end{array}$ & & & & & 460.8 \\
\hline $\begin{array}{l}\text { Oberarm } \\
\text { (Bs) }\end{array}$ & 631 & $\begin{array}{c}1404 \\
(38.2 \%)\end{array}$ & $\begin{array}{c}190.8 \\
(51.9 \%)\end{array}$ & $\left(\begin{array}{c}36 \\
(9.9 \%\end{array}\right)$ & & & & 367.2 \\
\hline $\begin{array}{l}\text { Unter- } \\
\text { schenkel(Ss) }\end{array}$ & 520.2 & $\begin{array}{c}165.6 \\
(52.9 \%\end{array}$ & $\begin{array}{c}882 \\
(28.2 \%)\end{array}$ & $\begin{array}{c}59.4 \\
(18.9 \%)\end{array}$ & & & & 313.2 \\
\hline
\end{tabular}

Am Oberarm erweist sie sich bei dem 7 monatigen Fetus erheblich größer als bei 6 monatigen.

Bei dem 8 monatigen Fetus ist die Haarzahl am Scheitel $(1427,2)$ am größten, dann kommen die Brust $(703,8)$, der Unterschenkel (684), Oberarm $(511,2)$; sie ist am geringsten am Rücken $(410,4)$.

Vergleicht man die Befunde des 7 und 8 monatigen Fetus miteinander, so ergibt sich, die Haarzahl bei dem 8 monatigen Fetus ausnahmslos an den untersuchten Körperteilen spärlicher als bei dem 7 monatigen ist. 
Die Haar- und Haargruppendichtigkeit bei den Mischlingsfeten

Tabell 7. 10-monatiger Mischlingsfetus (Japanerin $\times$ Europäer).

\begin{tabular}{|c|c|c|c|c|c|c|c|}
\hline \multirow{3}{*}{ Körp'rteile } & \multicolumn{7}{|c|}{ Haar- und Haargruppenzahl in $1 \mathrm{gcm}$ und Prozentsatz } \\
\hline & \multirow{2}{*}{ Haar } & \multicolumn{6}{|c|}{ Haargruppe } \\
\hline & & $1-\mathrm{HG}$ & $2-\mathrm{HG}$ & 3-HG & 4-HG & 5-HG & Summe \\
\hline Scheitel & 622.2 & $\left(\begin{array}{l}16.2 \\
(7.7 \%)\end{array}\right.$ & $\begin{array}{c}48.8 \\
(23.1 \%)\end{array}$ & $\begin{array}{c}86.4 \\
(41.0 \%)\end{array}$ & $\begin{array}{c}468 \\
(22.2 \%)\end{array}$ & $\begin{array}{l}12.6 \\
(5.9 \%)\end{array}$ & 2.0 .8 \\
\hline Brust & 350.6 & $\begin{array}{c}116.6 \\
(49.1 \%)\end{array}$ & $\begin{array}{c}117 \\
(50.9 \%)\end{array}$ & & & & 233.6 \\
\hline Rücken & 261 & $\left(\begin{array}{l}126 \\
70 \%)\end{array}\right.$ & $\begin{array}{c}45 \\
(25 \%)\end{array}$ & $\left(\begin{array}{l}9 \\
5 \%\end{array}\right)$ & & & 180 \\
\hline $\begin{array}{l}\text { Oberarm } \\
\text { (Bs) }\end{array}$ & 244.8 & $\begin{array}{c}59.4 \\
(40.3 \%)\end{array}$ & $\begin{array}{c}792 \\
(53.6 \%)\end{array}$ & $\left(\begin{array}{c}9 \\
(6.1 \%)\end{array}\right.$ & & & 147.8 \\
\hline $\begin{array}{l}\text { Unter- } \\
\text { schenkel(Ss) }\end{array}$ & 437.4 & $\begin{array}{c}73.8 \\
(31.8 \%)\end{array}$ & $\begin{array}{c}122.4 \\
(51.4 \%)\end{array}$ & $\begin{array}{c}39.6 \\
(16.8 \%)\end{array}$ & & & 235.8 \\
\hline
\end{tabular}

Bei noch einem 8 monatigen Mischlingsfetus zwischen Japanerin und Neger ist die Haarzahl auch am Scheitel $(1238,2)$ am größten, dann folgen die Brust $(705,6)$ der Rücken $(670,6)$, der Oberarm (631) und am niedrigsten ist sie am Unterschenkel $(520,2)$.

Aus obigen Tatsachen ersieht man, daß die Haarzahl am Scheitel, Rücken und Unterschenkel beim Mischlingsfetus zwischen Japanerin und Neger kleiner als bei dem zwischen Japanerin und Europäer ist.

Auch bei dem 10 monatigen Fetus nimmt der Scheitel $(922,2)$ die größte Haarzahl ein, dann folgẹn der Unterschenkel (437,4), Rücken $(350,6)$, die Brust $(261)$ und sie ist am Oberarm $(244,8)$ am kleinsten.

Aus den obigen einzelnen Befunden erkennt man die auffallende Tatsache, da $B$ nämlich die in $1 \mathrm{qcm}$ Hautflächeninhalt befindliche Haarzahl der verschiedenen Körperteile bei den untersuchten fetalen Mischlingsindividuen sich mit der Zunahme des Alters umgekehrt vollkommen parallelisiert, obwohl wenige Ausnahmen vorhanden sind. Mit anderen Worten, die Haare der verschiedenen Körperteile nahmen mit dem Fetalmonat nach und nach an Zahl ab. Diese Befunde stimmen mit den Befunden von Kato bei japanischen Feten vollkommen überein.

Die Gesamtzahl der verschiedenen Haargruppen in $1 \mathrm{qcm}$ Hautflächeninhalt.

Auch bei den fetalen Individuen sind die Haare einzeln oder zu Haargruppen implantiert. Nach meiner Untersuchung beläuft sich die Haarzahl einer Haargruppe auf 2-8 Haare. Jede Haargruppe ist von den benachbarten Haargruppen durch haarfreie $Z$ wischenräume getrennt. 
Also kann man die Haargruppenarten unterscheiden, d.h. zwei- bis achthaarige Haargruppen. Zwecks besserer Erklärung soll das einzelne Haar als eine Haargruppe, d.h. einhaarige Haargruppe, behandelt werden.

Die Haargruppenzahl in $1 \mathrm{qcm}$ Hautflächeninhalt bei verschiedenen Embryonalmonaten ist wie folgt.

Reihenfolge nach der Größe der Haargruppenzahl.

5 monatiger Fetus: Scheitel (1186,2), Brust (1089), Rücken (975,9), Oberarm (894,6), Unterschenkel $(724,5)$.

6 monatiger Fetus : Scheitel $(963,1)$, Rücken $(634,4)$, Unterschenkel (639), Brust $(626,4)$, Oberarm $(574,2)$.

7 monatiger Fetus: Brust $(608,4)$, Scheitel $(590,4)$, Oberarm $(583,2)$, Unterschenkel $(538,2)$, Rücken $(511,2)$.

8 monatiger Fetus: Scheitel $(484,1)$, Brust $(417,6)$, Oberarm $(320,4)$, Unterschenkel $(311,4)$, Rücken $(271,8)$.

9 monatiger Fetus (Misch. $z$ w. Japanerin und Neger): Scheitel $(568,8)$ Brust (460,8), Oberarm (367.2), Rücken (347,4), Unterschenkel $(313,2)$.

10 monatiger Fetus : Unterschenkel $(235,8)$, Rücken $(233,6)$, Scheitel $(210,8)$, Brust $(180)$, Oberarm $(147,6)$.

Wie aus den Tabellen leicht ersichtlich, ist die Haargruppenzahl der untersuchten 5 Körperteile bei dem 5 monatigen Fetus am höchsten, dann folgen der 6-, 7-, 8- und 10 monatige. Hieraus kann man den Schlu $\beta$ ziehen, da $\beta$ die Haargruppenzahl in den verschiedenen Körperteilen ausnahmslos mit der Zunahme das Alters regelmäßig sich vermindert.

Nach Kato ist die Haargruppenzahl am Scheitel immer größer als an sonstigen Körperteilen. Aber bei mir ist die Haargruppenzahl des Scheitels nicht immer am größten.

Die Reihenfolge der Haargruppenzahl nach Größe stimmt im großen und ganzen mit der der Haarzahl überein, woraus sich von selbst versteht, da $\beta$ die Haarzahl und Haargruppenzahl auf $1 \mathrm{qcm}$ Hautstück sich miteinander fast vollkommen parallelisiert. Nämlich an einem Körperteile, wo man eine große Haargruppenzahl findet, können auch reichliche Haare auftreten, obwohl nach den Individuen und Körperteilen einige kleine Schwankungen vorhanden sind.

\section{Die Zahl der verschiedenen Haargruppen in $1 \mathrm{qcm} \mathrm{Hautflächeninhalt.}$}

Wie schon erörtert, konnte ich auch bei den untersuchten Feten 8 Arten der Haargruppen, d. h. 1-8 haarige Haargruppen, unterscheiden. Die 1- und 2-haarigen Haargruppen befinden sich an allen untersuchten 
Körperteilen, und 3-haarige Haargruppe fand ich nicht am Unterschenkel (beim 5 monatigen Fetus), Oberarm (beim 6-monatigen Fetus), Rücken (beim 8-monatigen und 10-monatigen Fetus) und an der Brust (beim 8 monatigen Fetus, Japanerin-Negermischling). Die 4-haarige Haargruppe befindet sich nur am Scheitel, Unterschenkel und Oberarm. Die aus 5 bis 8 Haaren bestehenden Haargruppen kommen nur am Scheitel vor.

Beim 5-monatigen Fetus ist die Zahl der 1-haarigen Haargruppe wie in der Tabelle gezeigt, am Rücken (947) am größten, es folgen die Brust $(932,4)$, der Oberarm $(865,8)$, Unterschenkel $(707,5)$ und sie ist am geringsten am Scheitel $(572,4)$. Beim 6-monatigen Fetus ist sie am Unterschenkel $(448,2)$ am größten, dann folgen der Oberarm $(433,8)$, Rücken (260,1), die Brust (250,2) und der Scheitel (216). Beim 7monatigen Fetus ist ${ }^{\circ}$ die $Z$ ahl der Einhaargruppe auch am Unterschenkel (315) am größten, dann kommen die Brust $(219,6)$, der Rücken $(217,8)$, Oberarm (194,4) und der Scheitel (36). Beim 8-monatigen Fetus ist die Reihenfolge der Einhaargruppe wie folgt: Brust $(160,2)$, Oberarm $(158,4)$, Rücken $(133,2)$, Unterschenkel $(82,8)$ und Scheitel $(77,2)$. Beim 10-monatigen Fetus ist die Reihenfolge wie folgt: Brust (126), Rücken $(116,6)$, Unterschenkel $(73,8)$, Oberarm $(59,4)$ und Scheitel $(16,2)$.

Die obigen Befunde zeigen die bemerkenswerte Tatsache, daß die Zahl der Einhaargruppe in allen Fetalmonaten am Scheitel am kleinsten ist.

Die Reihenfolge der 2-haarigen Gruppe in der verschiedenen Fetalmonaten ist wie folgt:

5-monatiger Fetus: Scheitel (486), Brust (153), Rücken (27,1), Oberarm (19,8), Unterschenkel (18).

6-monatiger Fetus: Scheitel $(401,4)$, Rücken $(383,4)$, Brust $(374,4)$, Unterschenkel (151,2), Oberarm (1404).

7-monatiger Fetus: Brust $(340,2)$, Oberarm $(318,6)$, Rücken $(289,8)$, Scheitel $(137,8)$, Unterschenkel $(120,6)$.

8-monatiger Fetus : Brust, Rücken (138,6), Oberarm (136,8), Scheitel $(102,2)$, Unterschenkel $(84,6)$.

10-monatiger Fetus: Brust (126), Rücken $(116,6)$, Unterschenkel $(73,8)$, Oberarm $(59,4)$, Scheitel $(16,2)$.

Aus obigen Befunden geht hervor, daß, obwohl die Zahl der Zweihaargruppe nach dem Fetalmonat und den einzelnen Körperteilen verschieden groß ist, im großen und ganzen sie an der Brust viel größer ist.

Die Reihenfolge der 3-haarigen Gruppe ist wie folgt: 
5-monatiger Fetus: Scheitel (127,8), Oberarm (9), Brust $(3,6)$, Rücken (1,8), Unterschenkel (0).

6-monatiger Fetus: Scheitel (201,6), Unterschenkel $(39,6)$, Brust $(1,8)$, Rücken $(1,8)$, Oberarm $(0)$.

6-monatiger Fetus: Scheitel $(244,8)$, Unterschenkel $(100,8)$, Oberarm $(70,2)$, Brust $(48,6)$, Rücken $(3,6)$.

8-monatiger Fetus: Scheitel (176,4), Unterschenkel (144), Brust $(28,8)$, Oberarm (21,6), Rücken (0).

10-monatiger Fetus: Scheitel $(86,4)$, Unterschenkel $(39,6)$, Brust (9), Oberarm (9), Rücken (0).

Aus den oben erwähnten Befunden erkennt man Folgendes:

Bei der 3-haarigen Gruppe haben die Feten am Scheitel gegenfalls eine viel größere $Z$ ahl als an den sonstigen Körperteilen, wo immer eine kleine Haarzahl vorkommt.

Man kann auch sagen, da $\beta$ die Zahl der 3-haarigen Haargruppe mit Ausnahme des Scheitels mit dem Monatsalter nach und nach weniger wird.

Die Zahl der aus 4 bis 8 Haaren bestehenden Haargruppen ist, wie die Tabellen 2-7 zeigen, mit Ausnahme des Scheitels sehr gering, und die 5-, 6-, 7- und 8-haarige Gruppe lassen sich nur am Scheitel entdecken. Die 4-haarige Haargruppe kommt mit Ausnahme des Scheitels nur am Oberarm und Unterschenkel vor.

Am Scheitel konnte ich bei dem 5 -monatigen Fetus nur 3 Arten von Haargruppen, d.h. 1- bis 3-haarige, bei dem 6-monatigen und 7monatigen Fetus 7 Arten von Haargruppen vorfinden und bei dem 10 . monatigen Fetus kommen 1- bis 5-haarige Haargruppen vor. Beachtenswert ist, da $B$ beim 8-monatigen Fetus $z$ wischen Japanerin und Neger 1 - bis 5-haarige Gruppe, dagegen bei dem 8-monatigen Fetus zwischen Jananerin und Neger 1- bis 8-haarige Gruppe vorkommen. Doch kann man nicht diese Verschiedenheit als Rassenunterscheid annehmen, da das Material nur aus einem Fall besteht. Die Zahl der als mehr 4 Haaren bestehenden Haargruppen nimmt mit der zunehmenden Haarzahl nach und nach ab.

Der Prozentsatz der Zahl der verschiedenen Haargruppen zur gesamten Haargruppenzahl ist wie folgt.

Aus der Tabelle 2 erhellt sich, da $B$ der Prozentsat $z$ der einhaarigen Haargruppe beim 5-monatigen Fetus in allen untersuchten Körperteilen am größten ist, dann der der zweihaarigen und der dreihaarigen Haargruppen folgen. Beim 6-monatigen Fetus ist der Prozentsatz der 2haarigen Gruppe am Scheitel, Rücken und an der Brust am größten, 
dagegen am Oberarm und Unterschenkel der der 1-haarigen Gruppe am größten.

Beim 7 monatigen Fetus zeigt an der Brust, am Rücken und Oberarm die 2-haarige Gruppe den größten Prozentsatz. Am Scheitel zeigt die 3-haarige Gruppe den größten Prozentsatz und am Unterschenkel die einhaarige Gruppe den größten.

Beim 8-monatigen Fetus zwischen Japanerin und Europäer zeigt sich die 3-haarige Gruppe am Scheitel und Unterschenkel, die 2-haarige am Rücken und an der Brust und die 1-haarige am Oberarm den größt $\subseteq$.

Beim 8-monatigen Fetus zwischen Japanerin und Neger ist am Scheitel, Rücken, Oberarm und der Brust die $Z$ weihaargruppe prozentual am reichlichsten. Am Unterschenkel zeigt dagegen die Einhaargruppe den größten Prozentsatz. In bezug auf dem Prozentsatz der verschiedenen Haargruppen kann man sagen, $\mathrm{da} B \mathrm{z}$ wischen den beiden Mischlingen ein Unterschied annehmbar sei. Aber es läßt sich mit Sicherheit dieser Unterschied als eine Rassenverschiedenheit nicht annehmen.

Beim 10-monatigen Fetus ist der Prozentsatz der 3-haarigen Gruppe am Scheitel am größten. An Rücken, Oberarm und Unterschenkel ist der der 2-haarigen Haargruppe am höchsten und an der Brust die 1haarige Gruppe am reichlichsten.

Aus obigen Befunden kann man verstehen, daß die 2-haarige Haargruppe im ganzen Körper am gleichmäßigsten sich verteilt, ihr folgt die 1.haarige Gruppe.

Der Prozentsatz der aus mehr als 3 Haaren bestehenden Haargruppen ist, den Scheitel ausgenommen, im allgemeinen sehr niedrig und kleiner als 1\% (Oberarm, Unterschenkel). Beim dem 5-montigen Fetus findet sich keine dieser Haargruppen.

Am Scheitel ist der Prozentsatz bei dem 7 monatigen Fetus (29.3\%) am größten, dann folgen der 10 monatige $(28.1 \%)$ und 8 monatige Fetus (24.8\%), zuletzt kommt der 8 monatige Fetus (Mischling zwischen Japanerin und Neger).

Vergleicht man nun den Prozentsatz zwischen beiden 8 monat!gen Mischlingsfeten miteinander, so ist er beim Neger-Japanerin-Mischlingsfetus viel kleiner als beim Europäer-Japanerin-Mischlingsfetus.

Vergleicht man ferner zusammenfassend meine Befunde mit dem von Kato, so stimmen im großen und ganzen die beide Befunde überein.

Faßt man nun die Hauptresultate meiner Untersuchung zusammen, so ergibt sich folgendes. 
1. Die Haarbalgzahl in einem bestimmten Hautflächeninhalt schwankt sowohl nach der Körperteilen als auch nach den Fetalmonaten in hohem Maße. Sie ist in allgemeinen bei dem 6-monatigen Fetus am größten und vermindert sich nach und nach mit der Zunahme des Fetalmonates. Der Grad dieser Verminderung schwankt auch nach den Körperteilen.

2. Die Gesamtzahl der verschiedenen Haargruppen variiert auch nach den Körperteilen und den Fetalmonaten ziemlich. Sie ist bei dem 5-monatigen Fetus am größten und nimmt mit dem Fetalmonat ab.

3. Die Reihenfolge der Größe der Haarzahl der verschiedenen Körperteile steht mit der der Haargruppenzahl fast parallel. So sind die Haar- und Haargruppenzahl im Kopf reichlicher als im Rumpf und in der Extremität.

4. Bei den Feten konnte ich 8 Arten der Haargruppe, d. h. 1- bis 8-haarige Haargruppen unterscheiden. Die 1- bis 3-haarigen Gruppen befinden sich in meisten untersuchten Körperteilen, und die 4-haarige Gruppe fand ich am Scheitel, Oberarm und Unterschenkel, aber nicht an anderen Körperteilen. Die aus mehr als 5 Haaren bestehende Haargruppe konnte ich nur am Scheitel entdecken.

5. Die Verteilung der einzelnen Haargruppen ist je nach den Haargruppenarten verschieden. Die Einhaargruppe ist in der Extremität reichlicher als in den sonstigen Körperteilen. Die 2-haarige Gruppe verteilt sich im ganzen Körper gleichmäßig, aber die Dreihaargruppe ist am Scheitel am reichlichsten.

6. Die Zahl der einzelnen Haargruppen ist beim 6-monatigen Fetus am größten, und erst danach wird sie mit dem Fetalmonat nach und nach weniger.

7. Der Prozentsatz der Einhaargruppe zur gesamten Haargruppenzahl ist in der Extremität größer, dann kommt Rumpf, und ist kleiner am Scheitel.

8. Die 2-haarige Gruppe verteilt sich im ganzen Körper am gleichmäßigsten, dann folgt die 1-haarige Gruppe. Die Verteilung der 3haarigen und aus mehr als 3 Haaren bestehenden Haargruppen ist viel ungleichmäßiger.

9. Wegen des geringen Materials konnte ich einen sicheren Rassenunterschied nicht bestätigen. 


\section{Literatur.}

1. Taniguchi, T., und Shibayama, T., Die Dichtigkeit der Körperbehaarung bei einigen Rassen. Folia Anat. Japon. Bd. 13. 1935.

2. und - Die Dichtigkeit der Körperbehaarung bei den Japanern Folia Anat. Japon. Bd. 13. 1935.

3. Kato, S., Untersuchungen über die Haar- und Haargruppendichtigkeit bei den japanischen Feten. Folia Anat. Japon. Bd. 14. 1936.

4. Mogi, E., Untersuchungen über die Haardichtigkeit bei den Feten einiger Säugetierarten. Folia Anat. Japon. Bd. 15 (Ergänzungsband).

5. Yoshioka, K., Quantitative Untersuchung der Anhangsorgane der Haut bei einem Affen. Folia Anat. Japon. Bd. 14. 1936. 